prior to surgically induced ischaemic stroke results in a reduced infarct volume 70 hour later in wild type but not Nrf2 deficient mice, possibly as a consequence of enhanced Nrf2-mediated redox protection in the context of ischaemicreperfusion injury.

Materials and methods To further investigate mechanisms by which dietary sulforaphane affords neuroprotection, we measured acute blood flow changes during and after focal cerebral ischaemia in mice fed vehicle (corn oil) or sulforaphane at $5 \mathrm{mg} / \mathrm{kg}$ for 3 days. Ischaemic stroke was induced via the Longa procedure, employing a surgically inserted filament to transiently occlude the middle cerebral artery under stereotaxic anaesthesia. Ischaemia followed by $60 \mathrm{~min}$ of reperfusion resulted in a reproducible temporal lobe stroke. Transcranial laser speckle imaging (Moor FLIPI 2) was used to assess blood flow with high temporal $(20 \mathrm{~s})$ and spatial $(20 \mu \mathrm{m})$ resolution, enabling discrimination of three functional regions during stroke in the unaffected contralateral hemisphere, partially responsive per-infarct region and the ischaemic infarct core.

Results While sulforaphane did not alter initial baseline blood flow or affect the depth of ischaemia at the start of the procedure, a clear effect was resolved on the number of evoked peri infarct constriction (PIC) waves. While in both vehicle and treatment groups, an initial PIC was observed in all animals within $10 \mathrm{~min}$ of the onset of ischaemia, sulforaphane pre-treatment in wild type mice significantly reduced the number of subsequent PICs. In Nrf2 deficient mice, an equivalent and statistically significant reduction in PIC number was seen associated with sulforaphane pre-treatment, implying that the effects of sulforaphane on PIC frequency are unlikely to be mediated by Nrf2 but rather associated with associated redox gene induction. As sulforaphane significantly improved postreperfusion core blood flow in wild type not Nrf2 deficient mice.

Conclusions Our findings suggest that peri-infarct depolarisation waves or baseline Nrf2 genetic status are not major contributors to post-reperfusion, no-reflow in ischaemic stroke. However, activation of Nrf2 target genes by dietary sulforaphane protects against no-reflow pathology with persistent benefits post stroke.

Supported by the British Heart Foundation

\section{DEFINING THE NORMAL SPECTRUM OF ELECTROCARDIOGRAPHIC AND STRUCTURAL ADAPTATIONS OF WHITE, BLACK AND MIXED-RACE ADOLESCENT ATHLETES' HEART}

Aneil Malhotra, Sanjay Sharma. St. George's University of London and Hospital

\subsection{6/postgradmedj-2018-fpm.19}

Introduction This study aimed to describe the normal spectrum of EKG changes and left ventricular dimensions in a large cohort of healthy white, black and mixed-race, male and female adolescent soccer players. There are a paucity of data describing the adolescent athlete's heart with the vast majority of studies based on adult white athletes. Not only are adolescent athletes (aged 14-18 years old) significantly understudied compared with adults but are more vulnerable to sudden cardiac death than adults. There is a four to six-fold higher incidence of sudden cardiac death (SCD) during exercise among young black athletes (aged 14-35 years) compared with young white athletes. Such a significant disparity has never before been systematically explored, due to the limitations in identifying individuals at risk and a consequent lack of a well-defined population. The population of black individuals in the UK is 8 million, making up $13 \%$ of the overall population. In professional sport however, black athletes constitute up to $30 \%$ of all sportsmen and sportswomen. Moreover, the mixed-race athlete's EKG and echocardiogram has not before been described, though this is the fastest growing ethnic group participating in sport in the UK and US and for whom no such previous data exist.

Methods Between 1996 and 2016, 11435 consecutive adolescent soccer players underwent pre-participation screening. 11168 soccer players (mean age 16.4 1.3 years) were evaluated with an EKG and echocardiogram measuring maximal left ventricular wall thickness (MLVWT) and left ventricular end diastolic diameter (LVEDD). Ethnicity was self-reported in accordance with UK census classification.

Results The cohort included 10581 (95\%) males and 587 (5\%) females. There were 9664 (87\%) white, 1004 (9\%) black and 500 (4\%) mixed-race athletes.

Black male athletes exhibited a greater MLVWT compared with both white (10.9 vs. $10.6 \mathrm{~mm}$; $\mathrm{p}=0.0323)$. (Figure 1). White male athletes demonstrated the largest LV cavity sizes compared with black athletes $(52.4$ vs. $50.6 \mathrm{~mm}$; $\mathrm{p}=0.0477)$.

Black female athletes revealed a greater MLVWT compared with white female athletes ( 8.6 vs. $8.2 \mathrm{~mm}$; $\mathrm{p}=0.0352$ ). As with males, white female athletes demonstrated larger LV cavity sizes than black athletes $(46.3$ vs. $45.2 \mathrm{~mm} ; \mathrm{p}=0.0088)$ and mixed-race athletes (46.3 vs. $45.4 \mathrm{~mm}$; $\mathrm{p}=0.0316$ ).

For white males, a proposed upper limit for MLVWT is $13 \mathrm{~mm}$ and for black and mixed-race males $<14 \mathrm{~mm}$. For females of all three ethnic groups this cut off is $<11 \mathrm{~mm}$.
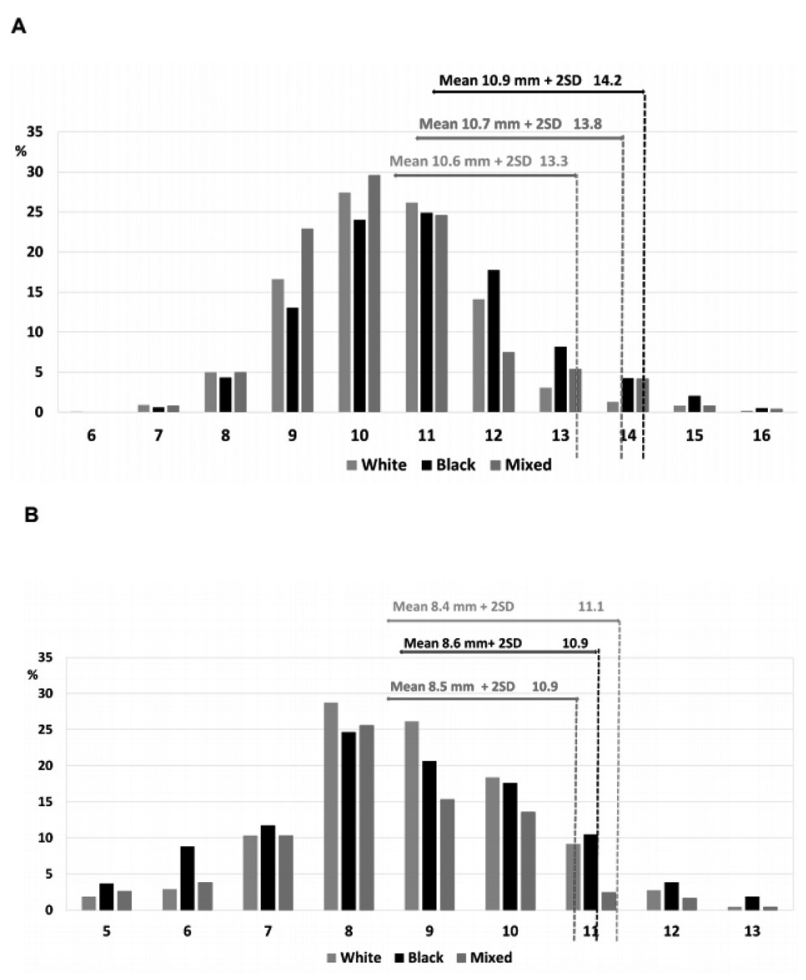

Abstract 8 Figure 1 Bar chart demonstrating the distribution of maximal left ventricular wall thickness in adolescent ( $A$, top) male and ( $B$, bottom) female athletes of white, black and mixed-race ethnicity. Upper limits according to 2 standard deviations (SD) are also shown 
Proposed upper limits for maximal LVEDD for white male is $<60 \mathrm{~mm}$ and for black and mixed-race males $<59 \mathrm{~mm}$. For all females the cut-off is $<52 \mathrm{~mm}$.

Conclusions Black athletes of both sexes reveal the greatest wall thickness compared with mixedrace and white athletes. Conversely, white athletes display the largest cavity size in both sexes. Mixed-race athletes demonstrate electrocardiogram and echocardiogram features more similar to black athletes than white athletes. Information regarding the upper limits of normality in this age group is useful in enabling the differentiation between physiological adaptation and cardiomyopathy.

\section{P62 DEFICIENCY PROTECTS AGAINST CEREBRAL ISCHAEMIA IN INSULIN RESISTANT MICE}

Siddall, Keith Farrell-Dillon, Paul Fraser, Giovanni Mann, Sarah Chapple. King's College London, UK

10.1136/postgradmedj-2018-fpm.20

Introduction and aim Diabetic patients have poorer outcomes following ischaemic stroke. In rodents, similar findings are reported, with Type 1 and 2 diabetic rodents demonstrating increased infarct volume and worsened post-stroke recovery, which correlate with markers of autophagy overactivation post-ischaemia. p62 is a selective adaptor protein for autophagy, with mice lacking p62 (p62 KO) known to develop obesity-linked insulin resistance in adulthood and have supressed autophagic flux. To ascertain whether p62 is associated with ischaemic stroke pathophysiology, we evaluated post-stroke infarct volume and functional recovery in wild type (WT) vs p62 KO mice.

Methods All procedures were performed in male 8-9 week old C57BL/6 mice in accordance with the ARRIVE guidelines and Home Office regulatory approval. Transient focal ischaemia was induced by middle cerebral artery occlusion (MCAo) for 1 hour followed by reperfusion for 72 hour. Adhesive removal and open field assays were conducted daily 3 days prior to and 72 hour post-stroke to assess sensorimotor and motor function. Mice were then euthanised and brains perfused-fixed to obtain $10 \mu \mathrm{m}$ sections every $1 \mathrm{~mm}$ relative to bregma. Infarct volume and astrocytosis were quantified using nissl staining and GFAP immunofluorescence, respectively. Data were analysed by unpaired student's t-test or 2-way ANOVA and Bonferroni post-hoc test as appropriate.

Results Infarct volume assessed by nissl staining showed a significant $(\mathrm{p}<0.01)$ reduction in $\mathrm{p} 62 \mathrm{KO}$ vs WT mice $(16 \pm 3$ vs. $30 \% \pm 1 \%$ brain volume, respectively). Proliferation and activation of astrocytes assessed by GFAP staining was found to be reduced in the peri-infarct border in p62 KO vs WT mice $(\mathrm{p}<0.05)$. Furthermore, p62 KO showed marked improvements in post-reperfusion functional recovery. Reduced motor function was observed in WT mice, with a $70 \%$ reduction in max speed and total displacement vs baseline observed 72 hour post-reperfusion $(\mathrm{p}<0.01)$. No such impairments were reported in p62 KO mice. Similarly, sensorimotor function was impaired in WT mice post-reperfusion, with an increase in adhesive removal time in the affected $(227 \pm 32$ vs $29 \pm 9 \mathrm{~ms}$ at baseline, $\mathrm{p}<0.001)$ and unaffected $(73 \pm 23 \mathrm{vs} 16 \pm 3 \mathrm{~ms}$ at baseline, $\mathrm{p}<0.05$ ) paw. p62 KO mice were protected, with adhesive removal times in the affected $(51 \pm 44$ vs $28 \pm 7 \mathrm{~ms}$ at baseline, $\mathrm{p}<0.05)$ and unaffected $(61 \pm 39$ vs baseline $28 \pm 7 \mathrm{~ms}$ at baseline) paws substantially improved when compared to WT mice at 72 hour.

Conclusions Our findings show that p62 KO mice are protected from cerebral ischaemia. This protection persists despite p62 KO mice being glucose intolerant, suggesting that p62 may be a meaningful therapeutic target even in comorbid diabetic populations. Further studies are needed to ascertain whether short-term functional recovery afforded by p62 deficiency persists long-term.

\section{EVIDENCE THAT ALTERED REDOX STATUS RESULTS IN KCA3.1 CHANNEL REDUCED ENDOTHELIAL CELL SURFACE EXPRESSION}

${ }^{1}$ Paul Fraser, ${ }^{1}$ Anas Bani Khalaf, 'Giovanni Mann, 'Matthew Smith, ${ }^{2}$ Geraldine Clough. ${ }^{1}$ King's College London; ${ }^{2}$ University of Southampton, UK

10.1136/postgradmedj-2018-fpm.21

Introduction Endothelial derived hyperpolarization $(\mathrm{EDH})$ is an important path to vasodilatation especially in small arteries, and is more important in women and the elderly. Our previous work has shown that this becomes impaired in mice that had experienced a high fat diet during gestation up to weaning, with concomitant reduction in endothelial surface KCa3.1 expression. ${ }^{1}$ We have tested the idea that altered redox status affects the location of $\mathrm{KCa} 3.1$ in human dermal microvascular endothelial cells (HDMECs).

Methods HDMECs were obtained from PromoCell (Heidelberg, Germany) and cultured in MV2 growth medium containing $1 \%$ penicillin-streptomycin in $5 \% \quad \mathrm{O}_{2}, 5 \% \mathrm{CO}_{2}$ at $37^{\circ} \mathrm{C}$ to passage 6 and 7 in the SCI-tive Physiological Oxygen Workstation from Baker Ruskinn (Maine, USA) for at least 5 days before experiments. The cells were plated on fibronectin (10 $\mu \mathrm{g} . \mathrm{ml}^{-1}$ ) coated 8 well micro-slides (Ibidi, Martinsried, Germany) and incubated for 48 hour, treated with PBS or $\mathrm{H}_{2} \mathrm{O}_{2} 100 \mu \mathrm{M}$ in PBS for $10 \mathrm{~min}$., and fixed with $4 \%$ formalin. Immunofluorescence was performed by incubating with anti-KCa3.1 mouse monoclonal antibody (AL-051 Alomone, Israel) at $4^{\circ} \mathrm{C}$ overnight, with half of the wells being treated with $2 \%$ Triton-X to permeablize the cells. The Alexa 488 labelled anti-mouse antibody for 1 hour and Texas Red Lycopersicon Esculentum Lectin (to stain the cell surface) for $20 \mathrm{~min}$, were applied to the cells at room temperature and finally the DAPI nuclear stain was applied for $3 \mathrm{~min}$. The cells were examined using a x60 1.3 NA oil-immersion objective and images captured via a Hamamatsu RG3 camera into a computer. Using ImageHopper software and computer-driven focussing device (Prior Instruments, UK) we took 10 serial images of $\mathrm{KCa} 3.1$ at $1.0 \mu \mathrm{m}$ intervals, which were integrated using Image J. These images were analysed for the fractional area of an endothelial cell occupied by $\mathrm{KCa} 3.1$, defined as bright spots between 1 to $4 \mu \mathrm{m}$ diameter in the relevant fluorescence channel.

Results The density of channels on the surface, defined as the integrated image of non-permeablized KCa3.1 channels, was $9.5 \pm 0.68 \mu \mathrm{m}^{-2} \times 10^{3}$ (mean \pm sem), while in the permeablized cells the density within the whole cell area was 13.9 $\pm 1.89 \mu \mathrm{m}^{-2} \times 10^{3}(\mathrm{p}<0.05$, ' $\mathrm{t}$ ' test, 4 experiments with 20 cells per experimental group) indicating that about $70 \%$ of the channels normally reside on the cell surface. Treatment with $\mathrm{H}_{2} \mathrm{O}_{2}$ had little effect on the total cell density (reduced 